\title{
REVALIDAÇÃO DE SARCOPROMUSCA TOWNSEND, 1927 COM REDESCRIÇÃO DE S. PRUNA (SHANNON \& DEL PONTE, 1926) (DIPTERA, MUSCIDAE, MUSCINAE) E CHAVE PARA OS GÊNEROS PRÓXIMOS
}

\author{
Denise Pamplona ${ }^{1,2}$
}

\begin{abstract}
The author revalidates the genus Sarcopromusca Townsend, 1927 including two species: S. pruna (Shannon \& Del Ponte, 1926) and S. sarcophagina (Wulp, 1896). The former is herein redescribed with illustrations and known geographical distribution. $A$ key to distinguish Morellra Robineau-Desvoidy, 1830, Orthellia Robineau-Desvoidy, 1830, Pyrellia Robineau-Desvoidy, 1830 and Sarcopromusca Townsend, 1927 is also presented.
\end{abstract}

\section{INTRODUÇÃO}

As espécies de Sarcopromusca são neotropicais e fazem parte de um grupo de Muscinae metálicos que inclui Morellia Robineau-Desvoidy, 1830 e Biopyrellia Townsend, 1932, assim como Orthellia Robineau-Desvoidy, 1830 e Pyrellia Robineau-Desvoidy, 1830 que não têm espécies neotropicais.

Pela análise dos caracteres filogenéticos dos Muscinae (Hennig, 1965), é difícil aproximar esta subfamília de qualquer outro grupo, devendo-se, portanto, considerá-la como grupo-irmão de todos os demais Muscidae s. str. restantes (com exceção dos Acanthipterinae).

Muscini, tribo a qual pertence, provavelmente monofilética, tem como caracteres apomórficos, pteropleura (anepímero) pilosa, arista plumosa e espiráculos posteriores das larvas sinuosos. Estes caracteres também existem nos Stomoxyinae, talvez por convergência (Hennig, 1965). Os Muscini foram aproximados aos Hydotaeini, pela presença do ovipositor longo e tubular, talvez relacionado com o modo de oviposição (Herting, 1957). Ovipositores compridos e delgados são bem adaptados para introduzir os ovos em substrato mole, o que pode ser observado em Sarcopromusca, que freqüentemente ovipõe em fezes de gado frescas. Este hábito também é facilitado pela presença de espinhos robustos, em forma de pentes, no VII e VIII segmentos do ovipositor de Sarcopromusca.

Sarcopromusca Townsend, 1927 tem sido considerado, até o presente estudo, como sinônimo de Morellia Robineau-Desvoidy, 1830 do qual se distingue pela presença dos seguintes caracteres: coloração geral enegrecida com polinosidade prateada; ápice do abdômen dourado; cerdas dorso-centrais $3+4$; acrosticais $0+2$; catepisternais $1: 3$; mero ciliado; bordo interno da caliptra ciliado; tíbia média na face ventral com 1 cerca préapical.

1. Universidade Federal do Rio de Janeiro/Museu Nacional/Depto. de Entomologia, Rio de Janeiro, 20942.

2. Bolsista do CNPq. 
Townsend (1927) apresentou uma chave na qual diferenciou Morellia de Sarcopromusca, pela presença nesta, de pélos no mero e cor levemente metálica e descreveu S. arcuate.

A diagnose genérica de Sarcopromusca foi apresentada por Townsend somente em 1937, onde incluiu comentários sobre a genitália do macho e da fêmea.

Chave para gêneros próximos:

1. Catepisternal anterior ausente. . . . . . . . . Biopyrellia Townsend, 1932 Catepistemal anterior presente . . . . . . . . . . . . . . . . 2

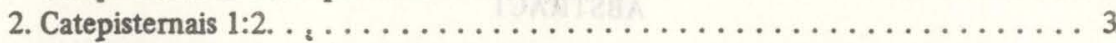

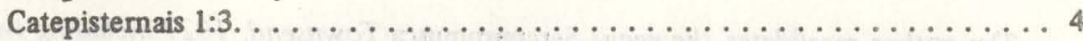

3. Bordo intemo da caliptra nu. Tíbia média sem cerda ventral. Nervura $M$ levemente curva em direção a $R 4+5 \ldots \ldots \ldots \ldots \ldots$ Morellia Robineau Desvoidy, 1830 Bordo interno da caliptra ciliado. Tíbia com cerda ventral. Nervura $M$ fortemente curva em direção a $R 4+5 \ldots \ldots \ldots \ldots \ldots$ Orthellia Robineau-Desvoidy, 1830

4. Bordo intemo da caliptra nu. Dorso-Centrais $2: 4-5$. Coloração geral metálica. . . . . . Pyrellia Robineau-Desvoidy, 1830

Bordo interno da caliptra ciliado. Dorso-centrais 3:4. Coloração geral enegrecida com polinosidade prateada............... Sarcopromusca Townsend, 1927

Sarcopromusca Townsend, 1927 nom rev.

Sarcopromusca Townsend, 1927: 208-209: Malloch, 1930: 480; Townsend, 1937: 56-57; Hennig, 1965: 36, 99.

Morellia; Pont, $1972: 9$ (partim).

Espécie-tipo: Orthelia pruna shannon \& Del Ponte, 1926 = Sarcopromusca arcuato Townsend, 1927. A este gênéro pertencem somente duas espécies: $S$. pruna (Shannon \& Del Ponte) e $S$. sarcophagina (Wulp).

\section{Sarcopromusca pruna (Shannon \& Del Ponte, 1926)}

Orthelia pruna Shannon \& Del Ponte, 1926: 22, 35 (holótipo fêmea, ARGENTINA; Jujuy, Calilègua - USNM).

Sarcopromusca arcuata Townsend, 1927: 209, 355-356 (holótipo fêmea, BRASIL: Itaquaquecetuba - USNM); parátipo fêmea, BRASIL:- Itaquaquecetuba - MZUSP; Malloch, 1930: 480-481; Sarcopromusca pruna; Shannon \& Del Ponte, 1928: 142, 146. Morellia pruna; Pont, 1972: 9.

Coloraçäo geral: Enegrecida com muita polinosidade prateada. Antena negra com polinosidade prateada. Parafrontália, parafaciália, faciália e gena com polinosidade prateada, ou dourada quando vistas sob certa iluminação. Palpo castanho. Tórax com quatro listras negras intercaladas de polinosidade prateada. Caliptras castanho-claras no macho e brancas na fêmea. Halter amarelado. Pernas castanho-escuras. Asa hialina. Abdômen com manchas negras e, tergito $\mathrm{V}$ dourado.

Machos: $6,0-7,0 \mathrm{~mm}$.

Cabeça: Olhos separados por um espaço de bordos divergentes para o vértice. Cerdas frontais com cerca de 14 pares ciliformes. Triângulo ocelar com um par de cerdas e vários culios. 
Tórax: Cerdas dorso-centrais $3+4$; acrosticais $0+2$; pós-pronotais 1 ; intra-alares $1+1$; supra-alares $1+3$; notopleurais 2 ; pós-alares 2 ; cerda intra-pos-alar 1 . Bordo interno da caliptra ciliado. Escutelo com um par de cerdas basais, um par de apicais e um par de discais. Proepímero largo e ciliado. Anepistemo ciliado com uma cerda no ângulo ântero-superior e cerca de 7 cerdas no bordo posterior, de tamanhos decrescentes (a superior maior). Catepisterno ciliado e com cerdas 1:3. Fêmur anterior nas faces ântero-ventral e ântero-dorsal com uma fileira de cerdas. Tíbia anterior com uma cerda dorsal pré-apical. Fêmur médio nas faces ventral e ântero-ventral com uma fileira de cerdas, face pósteroyentral na metade apical, com cerdas curtas que se continuam em direção a face postetior aumentando de tamanho. Tíbia média na face ventral com uma cerda pré-apical, na face posterior com duas cerdas no terço basal e duas no terço médio. Fêmur posterior na face ântero-vẹntral com uma fileira de cerdas, maiores na metade apical, face ântero-dorsal com uma fileira de cerdas e face póstero-ventral com cerca de 4 cerdas na metade basal. Tíbia posterior na face ântero-ventral com 4 cerdas, face ântero-dorsal com uma fileira de cerdas curtas e fortes, face dorsal com uma pré-apical e face póstero-dorsal com đuas cerdas no terço médio. Asa, $R 4+5$ na face ventral com alguns cílios no nódulo e face dorsal com cílios do nódulo até a metade da distância do nódulo até r-m.

Abdômen: Com muitos cílios de revestimento. Tergitos III, IV e V com cerdas na margem posterior.

Genitália: Cercos com incisão posterior bem profunda, projeção lateral presente (fig. 1a, 1b); sustilus com cerdas medianas na face interna, duas cerdas no terço basal da face externa e algumas cerdas curtas apicais (fig. 2); quinto esternito com incisão posterior arredondada (fig. 4); apódema do edeago com ápice arredondado ultrapassando um pouco o hipândrio, gonópodos longos e com várias cerdas basais e parâmeros com várias cerdas externas (fig'. $3 \mathrm{a}, 3 \mathrm{~b}$ ).

Material dissecado: 1 macho (MNRJ), Minas Gerais, Serra do Caraça, IV/1969, F. M. Oliveira col.

Fêmeas: $6,0-7,0 \mathrm{~mm}$.

Semelhantes aos machos diferindo no que se segue:

Olhos separados por um espaço de bordos paralelos. Cerdas frontais em núme ro de 12 pares. Fêmur médio com cerdas mais fracas. Tíbia média na face ântero-ventral com 3 cerdas.

Genitália: Epiprocto triangular com pequenos espinhos no ápice (fig. 6a). Hipoprocto arredondado (fig. $6 \mathrm{~b}$ ).

Material dissecado: holótipo (USNM, n. 40812).

Material examinado: ARGENTINA, Jujuy, Calilegua, holótipo fêmea (NMNH-SI, tipo 40812), 5/V/1926, Shannon \& Shannon col.; BRASIL: Minas Gerais, Serra do Caraça, 1 macho (MNRJ), IV/1969, F. M. Oliveira col.; Rio de Janeiro, Itatiaia, $800 \mathrm{~m}$, 1 fềmea (MNRJ), II/1933, H. S. Lopes e R. Cunha col., Itatiaia, Lago Azul, 1 feemea (MNRJ), 19-21/VI/1955, D. de O. Albuquerque, R. Barros e Pearson col.; São Paulo, Itaquaquecetuba, 1 fêmea (Townsend Collection) (NMNH-SI), 23/XII; 1 paratipo fêmea (n. 2208) (MZUSSP), 4/II, em folhagem; Campos do Jordão, 1 fêmea (MNRJ), XI/1936, J. Lane col. gentina.

Distribuição geográfica conhecida: Guatemala, Nicarágua, Costa Rica, Brasil, Ar-

Dados biológicos: Esta espécie é um muscídeo sinantrópico, importante veiculador de ovos de Dermatobia hominis (L.JR., 1781) (berne) no Estado do Rio de Janeiro, sendo sua biologia pouco estudada. 


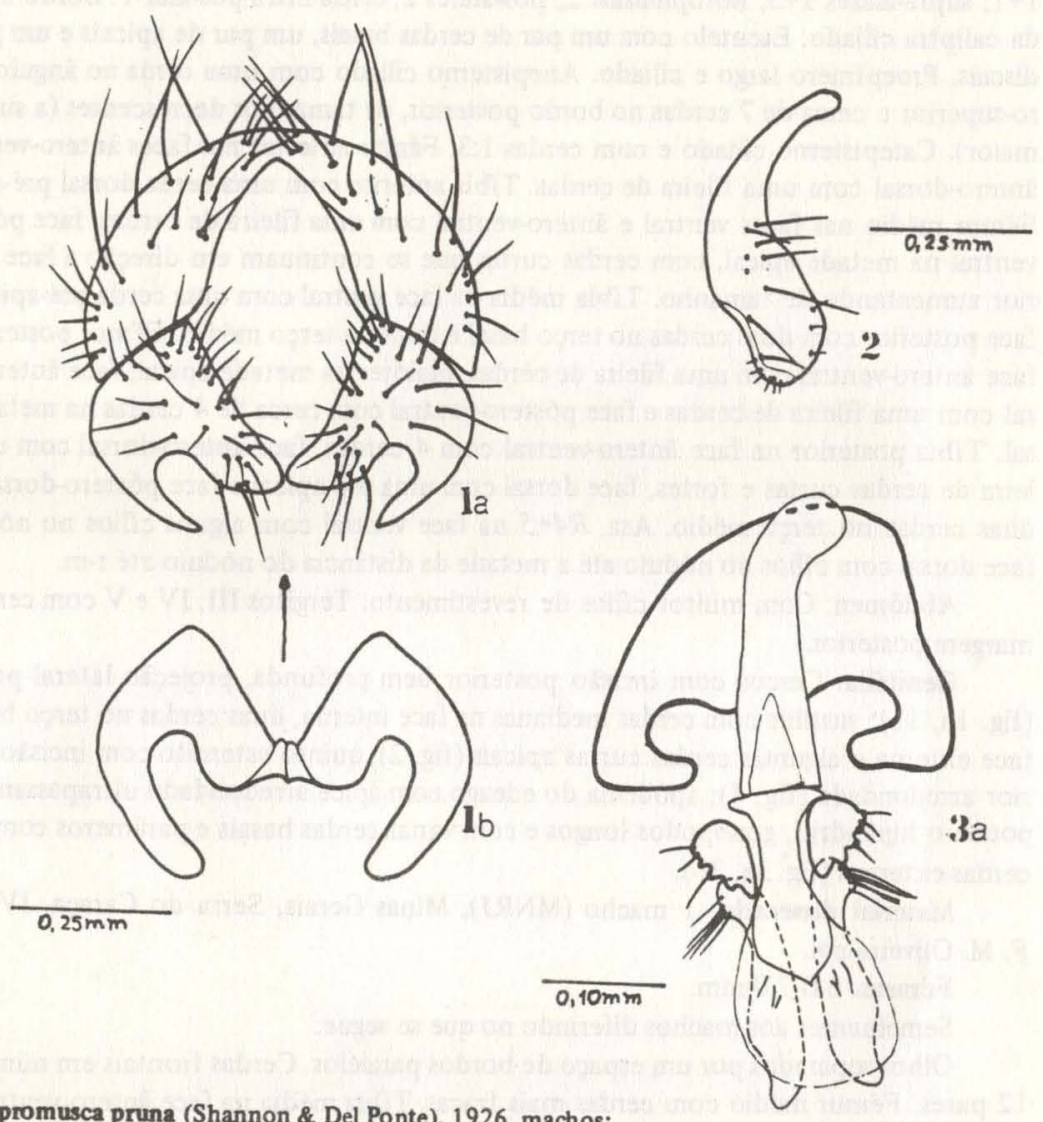

Sarcopromusca pruna (Shannon \& Del Ponte), 1926, machos:

Fig. 1a: epândrios e cercos, vista dorsal;

Fig. 1b: epândrios e cercos, vista ântero-dorsal;

Fig. 2: sustilo esquerdo, vista dorsal;

Fig. 3a: complexo fálico, vista ventral;

Sarcopromusca sarcophagina (Wulp, 1896) n. comb.

Morellia sarcophagina (Wulp, 1896): 302 (Sintipos: 2 machos, Atoyac, Vera Cruz, Teapa, Tebasco, MÉXICO - BMNH); Engel, 1931:134; Séguy, 1935: 113; Townsend, 1935: 190; Séguy, 1937: 395; Stein, 1918: 147; Stein, 1919: 109; Pont, 1972: 9.

Pelos dados da bibliografia consultada, os caracteres mais importantes de $S$. sarcophagina, que a diferenciam de $S$. pruna são: 1 . presença de mancha castanha ao longo da nervura costal; 2. tergito abdominal IV também dourado.

Distribuição geográfica conhecida: MÉXICO. 


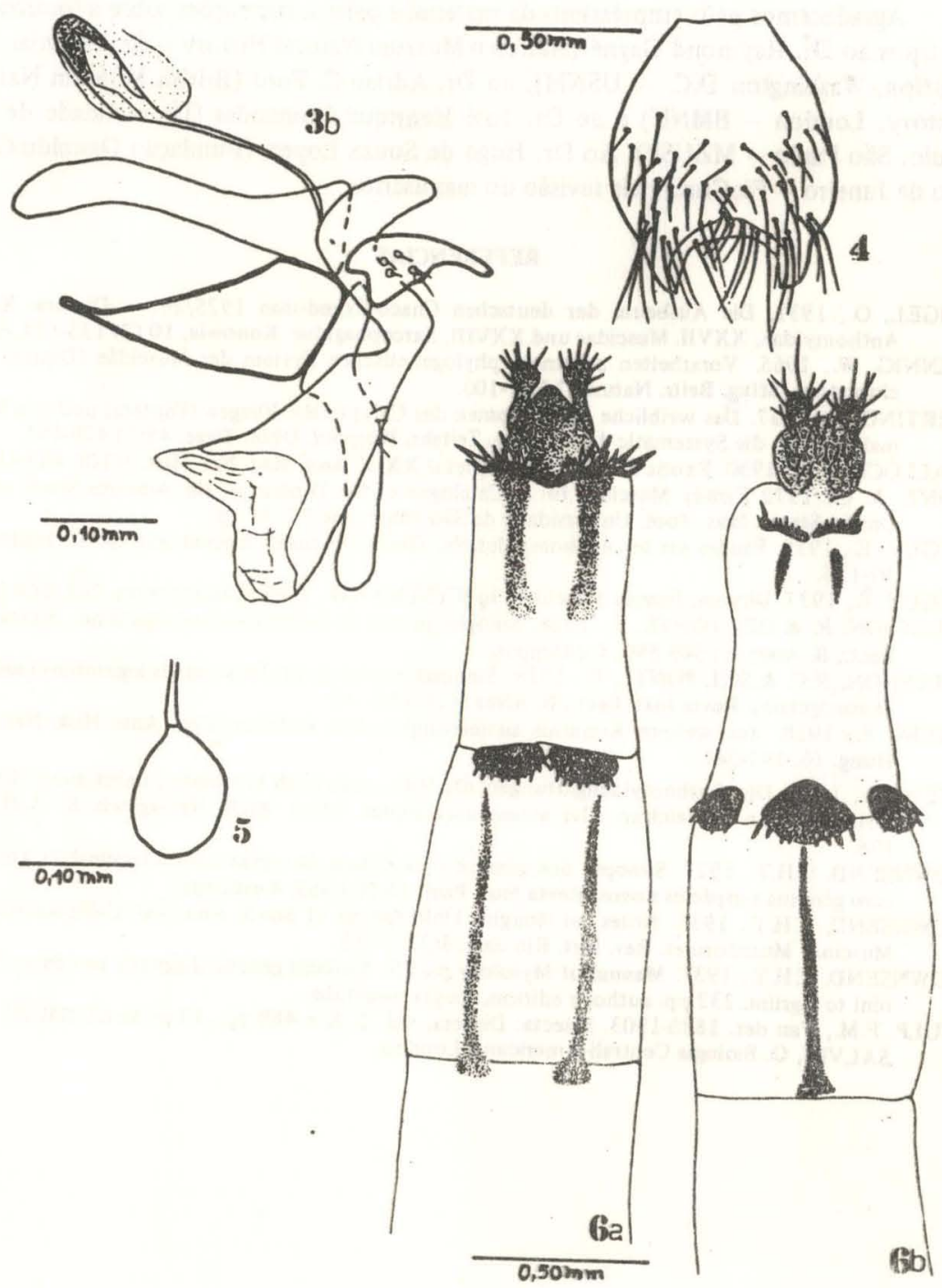

Sarcopromusca pruna (Shannon \& Del Ponte), 1926

\section{Macho:}

Fig. 3b: complex o fálico, vista lateral;

Fig. 4: quinto esternito, vista dorsal;

Fểmea:

Fig. 5: espermateca.

Fig. 6a: ovipositor, vista dorsal;

Fig. 6b: ovipositor, vista ventral. 


\section{AGRADECIMENTOS}

Agradecemos pelo empréstimo de material e pelas informações sobre a localização de tipos ao Dr. Raymond Gagné (National Museum Natural History - Smithsonian Institution, Washington D.C. - USNM), ao Dr. Adrian C. Pont (British Museum Natural History, London - BMNH) e ao Dr. José Hengrique Guimarães (Universidade de São Paulo, São Paulo - MZUSP), Ao Dr. Hugo de: Souza Lopes, (Fundação Oswaldo Crụz, Rio de Janeiro - FioCruz) pela revisão do manuscrito.

\section{REFERENCIAS}

ENGEL, O., 1931. Die Ausbeute der deutschen Chaco-Expedition 1925/26-Diptera. XXVI. Anthomyidae, XXVII. Muscidae und XXVIII. Sarcophagidae. Konowia, 10 (2):133-154, $6 \mathrm{Taf}$.

HENNIG, W., 1965. Vorarbeiten zu einem phylogenetischen System der Musciđăe (Diptera: Cyclorrapha), Sttug. Beitr. Naturk. 141:1-100.

HERTING, B., 1957. Das weibliche Postabdomen der Calyptraten Fliegen (Diptera) und sein Merkmalswert fur die Systematic der Gruppe. Zeitshr: Morphol. Okol. Tiere, 45(5):429-461.

MALLOCH, J. R., 1930. Exotic Muscaridae (Diptera) XXIX. Ann. Mag. Nat. Hist. 5(10): 465-484.

PONT, A. C, 1972 Family Muscidae In: A Catalogue of the Diptera of the America South of the United States. Mus. Zool. Universidade de São Paulo, vol. 97,11 pp.

SÊGUY, E., 1935. Etudes sur les Anthomyides, 9e, 10e et 11 e notes. Encycl. ent. (B.II) Diptera, 8: 97-116.

SEGUY, E., 1937. Diptera, Family Muscidae. In WYSTMAN, P., Genera Insectorum, 205, 604 pp.

SHANNON, R. \& DEL PONTE, E., 1926. Sinopsis parcial de los Muscoideos argentinos. Revta Inst. Bact., B. Aires (5):549-590, 4 estampas.

SHANNON, R.C. \& DEL PONTE, E., 1928. Sinopsis parcial de los Muscoideos argentinos (addenda et corrigenda). Revta Inst. Bact., B. Aires 5(1): 141-147.

STEIN, P., 1918. Zur weitern Kenntnis aussereuropåischen Anthomyiden. Ann. Hist. Nat. mus. Hung. 16: 147-244.

STEIN, P., 1919. Die Anthomyidengattungen der Welt, analytisch bearbeitet, nebst einem kritishsystematischen Verzeichnis aller aussereuropäischen Arten. Arch. Naturgesch. 83 A.(I): 85 178.

TOWNSEND, C.H.T., 1927. Synopse dos gêneros muscoideos da regiâo úmida tropical da América, com gêneros e espécies novos. Revta Mus. Paul. $15: 203-385,4$ estampas.

TOWNSEND, C.H.T., 1935. Notes on Hough's 1900 species of South American Calliphorinae and Muscinae Musciformes. Rev. Ent. Rio de J. 5(3): 1-366.

TOWNSEND, C.H.T., 1937. Manual of Myiology part V. Muscoid generic diagnosis and data, Glossinini to Agriini, $232 \mathrm{pp}$. author's edition, Itaquaquecetuba.

WULP, F.M. Van der, 1888-1903. Insecta: Diptera, vol. 2, X + 489 pp., 13 pl. In GODMAN, F.D.; SALVIN, O. Biologia Centrali-Americana, London. 